\title{
An Improved Image Compression- Decompression Technique Using Block Truncation and Wavelets
}

\author{
Kuldeep Mander \\ Computer Science and Engineering Department, Bahra Group of Institutes, Patiala, Punjab, India - 147001 \\ Email: kuldeepmander12dec@gmail.com \\ Himanshu. Jindal \\ Computer Science and Engineering Department, Thapar University, Patiala, Punjab, India - 147004 \\ Email: himanshu.jindal@thapar.edu
}

Received: 20 March 2017; Accepted: 08 May 2017; Published: 08 August 2017

\begin{abstract}
In the modern world, digital images play a vital role in a number of applications such as medical field, aerospace and satellite imaging, underwater imaging, etc. These applications use and produce a large number of digital images. Also, these images need to be stored and transmitted for various purposes. Thus, to overcome this problem of storage while transmitting these images, a process is used, namely, compression. The paper focuses on a compression technique known as Block Truncation Coding (BTC) as it helps in reducing the size of the image so that it takes less space in memory and easy to transmit. Thus, BTC is used to compress grayscale images. After compression, Discrete Wavelet Transform $(D W T)$ with spline interpolation is applied to reconstruct the images. The process is suggested in order to view the changed pixels of images after compression of two images. The wavelets and interpolations provide enhanced compressed images that follow the steps for its encoding and decoding processes. The performance of the proposed method is measured by calculating the PSNR values and on comparing the proposed technique with the existing ones, it has been discovered that the proposed method outperforms the most common existing techniques and provides $49 \%$ better results in comparison with existing techniques.
\end{abstract}

Index Terms-Compression; BTC (Block Truncation Coding); DWT (Discrete Wavelet Transform); Interpolation; Spline; Contrast.

\section{INTRODUCTION}

A digital image is a collection of pixels that has particular location and value. These images can be represented in mathematical form. These are also known as bitmap images or raster images. The demand of digital imaging is growing rapidly due to its applications in a large number of fields. But, digital images have their limitations that they take more space in memory and therefore take more time to transfer from one device to another. In such cases, the size of the image is of utter importance, so that better results can be produced. In this context, various compression techniques are present that include:

Lossy Compression: It is a compression technique in which there is some data loss on the receiver side which may not be noticed by the user. Various lossy compression techniques are-

i. Transform Coding: This technique works by considering the image pixels, then Discrete Cosine Transformation $(D C T)$ is applied upon them. The bits are allocated to encode them and after transmission the bits are decoded. The inverse of $D C T$ is applied to attain the output image.

ii. Fractal Compression: This technique divides the image into segments and uses the Quad tree coding followed by quantization.

iii. Vector Compression: It uses a code book on both sides to encode and decode the image.

iv. Block Truncation Coding: In this method, the image is divided into blocks and each block is reconstructed, quantized and finally reverse is carried out to get a good quality image.

v. Chroma Sub-Sampling: This method represents red, green or blue are represented to an intensity, initially and then reverse of this process is used to get an image.

Dossless Compression: The compression techniques under this category do not suffer from any data loss problems in an image. Some lossless compression techniques are -

i. Run Length Encoding $(\boldsymbol{R} L E)$ : In this compression technique, there is a sequence of repeating data in the file. This sequence or run is replaced by a single data and a count value. In $R L E$ larger values are replaced by smaller values. 
ii. Entropy Encoding $(\boldsymbol{E E})$ : In this type of compression technique, each incoming symbol is replaced by a fixed length code word. These code words are short in length and assign to symbols based on the probability of the symbol. This technique is a logarithmic technique.

iii. Huffman Coding: This technique is mostly used for text, images, videos and conferences. This technique selects symbols from the source image and calculate probability for each symbol. The calculation is based on the probabilities of image and a binary tree is generated. To construct binary tree, zeroes are assigned to left nodes and ones to the right node. At the end, all zeroes and ones are collected to generate code.

iv. Arithmetic Encoding: This technique is used to calculate the probability of the symbols like other techniques. The arithmetic encoding assign codes to each symbol based on the occurrence of that particular symbol.

v. LZW encoding: This technique assigns codes to symbols based on their occurrence following the $A S C I I$ table. In $L Z W$, a dictionary is generated for encoding and decoding, on both sides of the transmission module.

Out of these compression techniques, the paper focuses on Block Truncation Coding (BTC) as it provides the quantized reconstructed image in a very short time. It has the advantages of processing enhanced images when combined with Discrete Wavelet Transform (DWT) and interpolation. The proposed methodology of $B T C$ with $D W T$ and interpolation is explained by considering the existing studies of few researchers.

The organization of the paper is as follows. Section II discusses the literature survey carried out by researchers. Section III describes the preliminary studies of $B T C$, $D W T$. The proposed algorithm and results are discussed in Section IV. The proposed technique's comparison is shown in Section V. Finally, Section VI draws the main conclusion.

\section{RELATED WORKS}

The existing researches in image compression are discussed as under.

A. K. Katharotiya et al. [1] has explained techniques of compression like DCT and DWT. The author also explains these techniques by using various parameters such as compression ratio, the MSE value of the original image and compressed image by using DCT and DWT techniques.

A. Kaur and J. Kaur [2] has proposed image compression techniques $D C T$ and $D W T$, including the comparison of these techniques by using various parameters like compression ratio, PSNR, MSE, SER and time, etc. According to their comparison $D W T$ is better as compare to DCT technique.
A. Kaushik and M. Gupta [3] has proposed image compression, which is of lossy and lossless and also explained the process of compression and decompression. This technique includes very low frequencies do high frequency can be easily discarded without any loss of quality.

A. Sinha et al. [4] has presented an interpolation method which is good as compared to existing algorithms of interpolation. Bi-cubic interpolation is suitable for 3-D images and medical use, but for general application bilinear interpolation is used.

B. S. Kumar et al. [5] have proposed Image Enhancement approach on the basis of interpolation, $D W T$ and $S W T$. The application of the proposed strategy on some renowned images and the PSNR results prove this technique to be dominant over other conventional methodologies.

C. Sun et al. [6] has described a method to improve the contrast of an image. This method is dynamic contrast enhancement histogram equalization. It keeps same shape features and enhances contrast dynamically without losing the characteristics of original histogram. Dynamic histogram specification algorithm is used for this purpose. Due to its simplicity, it is used in real time systems. Noise effects are reduced using this method. On the other hand, $H E$ and $B P B H E$ are also used for contrast improvement, but it highlights the noise effects. Due to this limitation, humans do not accept these methods for contrast enhancement. To improve these limitations, a dynamic histogram specification algorithm is proposed. In many electric devices such as digital camera, mobile phone and $L C D$ panel, $D H E$ is also helpful to enhance contrast of the picture.

G. M. Padmaja and C. H.. R. Lakshmi [7], has represented the compression procedure. The DWT method is explained. In this paper where low pass and high pass filter are used to differentiate frequencies of two different types of frequencies, and in next step column wise filtering is done. For pyramid decompression; $L L$ bands are having the highest priority and is most useful instead of this technique, various other techniques are available like vector quantization, Entropy coding and arithmetic coding, SPIHT etc.

G. Rampani [8], has discussed an interpolation technique which changes the traditional operations of the existing techniques like bilinear, bi-cubic, and cubic spline into space variant techniques. This technique takes less computational time as compare to other techniques.

$H$. Hauang et al. [9] has designed a new grid interpolation algorithm. The existing methods of grid interpolation have the drawbacks of long back searching. The author has presented faster searching capacity algorithm J-nearest neighbor searching strategy is based on "priority queue" and "neighbor lag" concepts.

Hitashi et al. [10] has discussed about fractal compression technique. This technique is similar forms and patterns occurring in different sizes make a structure, e.g., a floor which is made up of either wood, tiles or concrete, but having repeating patterns in its texture if we compare all parts of the floor then we find various 
mathematical equations. These equations are known as fractal code. This fractal code describes the fractal properties or features of the pattern. The image can be regenerated by using these fractal equations.

M. Kaur and G. Kaur [11] has represented various lossless compression techniques like RLE, E.E, H.E and $L Z W$ are coding and various lossy compression techniques like T.C, DCT, DWT and F.C. and represent that lossy compression techniques provide more efficient compression as compare to lossless compression techniques. Lossless compression is used identical original image is required.

M. Tonge et al. [12] has studied various digital watermarking techniques. The Discrete Wavelet Transform (DWT) digital watermark algorithm based on human vision characters is proposed and implemented. The simulation result reveals that this watermarking system not only can keep the image quality, well, but also can be robust many common image processing operations of filter, etc. They had compared the results of the proposed technique with the reference method on the basis of evaluation parameters such as Normalized Cross Correlation $(N C)$ and Peak Signal-to-Noise Ratio (PSNR).

$M d$. F. Hossain and M. R. Alsharif [13] has proposed a new method, which is based upon the logarithmic transform coefficient adaptive histogram equalization, for image enhancement. On the basis of properties of logarithmic transform domain histogram and constraint limited adaptive histogram equalization, this method is performed. In addition, it is used $E M E$ as a measure of performance. This algorithm is compared to a classical histogram equalization enhancement technique on the basis of performance. The powerful method is LTAHE for enhancing images. The advantage of this method is that it is simply based on transform adaptive histogram. The results of this technique are outperformed as compared to commonly used enhancement technique like histogram equalization.

$R$. Olivier and C. Hanquing [14] has presented a new interpolation algorithm which is based on the nearest neighbor interpolation algorithm. But it uses the nearest values rather than distance to calculate the missing pixels.

Z. Min et al. [15], has presented an image zooming method which is based on the boundary effects on that image. This proposed technique is a combination of both nearest neighbor interpolation and Bi-linear interpolation. The proposed method is suitable for both types of images containing words and non-words information, e.g Posters, Books, etc. AEE is good and fast method as compared to the existing methods which are compared by using two parameters $M S E$ and $P S N R$ values. According to the $A E E$ method when unknown pixels are calculated by using nearest neighbor interpolation is coming under neighbor region and which is to be calculated by using bi-linear method is considered under bi-linear region.

In summary, there are approaches towards the compression of images, but existing approaches have their limitations such as (i) existing methods are not robust, (ii) methods are time consuming, (iii) provide distorted images and are noisy. Therefore, in order to remove these limitations to a certain extent, the paper focuses on proposing an enhanced compression technique in combination with Block Truncation Coding (BTC), $D W T$ and interpolation. The proposed technique compresses using $B T C$. To get decompressed images the process will go wither by the inverse of a $B T C$ or by $D W T$ and interpolation. The inverse of $B T C$ provides a decompressed image with distortion. Therefore, the paper suggests to use $D W T$ with interpolation to decompress images. Moreover, it provides good quality images in order to visualize newly formed pixels. The quality of images is determined by $P S N R$ values. The preliminary studies of the proposed method are discussed in the next section.

\section{PRELIMANARIES}

Before working on the proposed approach, the various existed approaches are explained by its working in the subsections. The proposed method uses Block Truncation Coding (BTC) for compressing images, Discrete Wavelet Transform (DWT) to improve the quality of images as $D W T$ provides functionality to decompose the image into sub-bands by virtue the user can easily correct the blurriness, noise present in images. The methodology for $D W T$ is combined with spline interpolation in order to find the newly formed pixels after correcting images (making images free from noise, blurriness). The working of $D W T$ and spline interpolation is explained as under.

\section{A. Block Truncation Coding (BTC)}

BTC algorithm uses $\mathrm{N}$ levels quantization process to quantize the image parts. It is a lossy compression algorithm. This is based on movement preserving quantization. The movement preserving means to retain the quality of the image and also, the storage space required that can be reduced. BTC algorithm follows three steps, i.e., (i) Performing the quantization, (ii) Coding the quantization and (iii) Bit plan reduction.

The standard mean and standard deviation are used to express the quantized data values of an image. The standard deviation represents the changes in the quantity and standard mean represents the average value of the pixels of a block. In BTC each block is encoded separately so it is beneficial to compress images having different types of blocks, e.g., text block, picture block and smooth block. In all these blocks there are various differences. The main advantages of using BTC are:

i. $\quad B T C$ is less complex.

ii. It is able to regenerate every edge of the original image.

iii. By using the variance of each block it can be compressed separately.

iv. It may be fixed or adaptive.

v. BTC requires little memory space.

vi. This is easy to implement.

\section{B. Discrete Wavelet Transform (DWT)}


In $D W T$, two types of filters are applied on the available frequency. These two types of filters are low pass filter and high pass filter. These filters divide the available frequency in exactly two halves. And process is applied onto each row, then to each column and collect their own frequencies from the available frequency. So, the filtering process results into two dimensional array of coefficients. This array consists of four bands low-low, high-low, low-high and high-high. Low-low band is further decompose into four bands to attain its second level decomposition. The low-low band is used for decomposition as it is easy to process. Moreover, the lowlow filter has highest importance as compared to all other bands. The following figures show two level view $D W T$.



Fig.1. First level decomposition of $D W T$

\begin{tabular}{|c|l|l|}
\hline $\mathrm{LL}_{2}$ & $\mathrm{HL}_{2}$ & \multirow{2}{*}{$\mathrm{HL}_{1}$} \\
\hline $\mathrm{LH}_{2}$ & $\mathrm{HH}_{2}$ & \\
\hline $\mathrm{LH}_{1}$ & & $\mathrm{HH}_{1}$ \\
& & \\
\hline
\end{tabular}

Fig.2. Second level decomposition showing sub-bands of $D W T$

The $D W T$ provides better quality images to know the unknown or newly formed pixels and for decompressing images using interpolation. Following steps shows the encoding and decoding process of DWT. Image decomposition by Discrete Wavelet Transform.

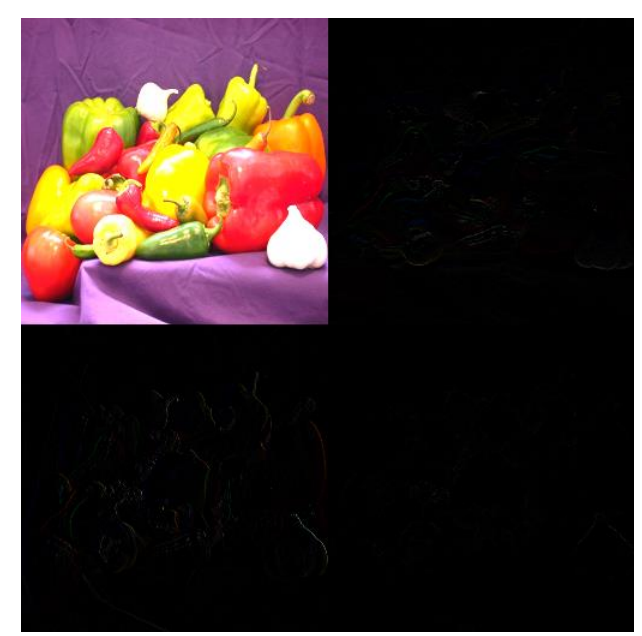

Fig.3. Output pepper image of $D W T$
This technique includes very low frequencies do high frequency can be easily discarded without any loss of quality. There are various interpolation techniques that include spline, linear, bi-linear, cubic, bi-cubic and nearest neighbor interpolation. Out of these, spline outperforms better and is discussed in the next subsection.

\section{Spline Interpolation}

The spline interpolation is used to approximate the unknown pixel data for a given image to enhance the size of the given image. The new points are determined with respect to some known points. The spline takes into consideration, a larger domain of pixels in order to produce better interpolation results. This technique is faster and provides zoomed images of better quality.

\section{Proposed AlgorithM AND EXPERIMENTAL RESUlTS}

The image compression technique involves the application of BTC on the input images and the PSNR values are calculated. The DWT with 'haar' wavelet is applied on produced output by BTC to attain four sub images and are further processed by the interpolation of these sub-images to reconstruct the image and finally, inverse of $D W T$ is applied to attain the final output image. The algorithm for the process is explained as under:

\section{Algorithm 1: Improved Block Truncation Coding Algorithm} 1: start

2: $\quad$ A $\leftarrow$ image

3: apply BTC (A) // block truncation code technique is applied in the input image.

4: calculate $P S N R$ (A, X) //finding PSNR for compressed an original image.

5: $\quad$ Decompose ' $X$ ' into four sub-bands say (a1, a2, a3, a4) using $D W T$ with 'haar' wavelet

6: for $\mathrm{i}=1, . ., 3 / /$ differentiating $R, G, B$ colors of each of 4 sub-bands

7: $\quad$ b $[\mathrm{i}] \leftarrow \mathrm{a} 1(:, ;$,i $)$

8: $\quad c[i] \leftarrow a 2(:,:, i)$

9: $\mathrm{d}[\mathrm{i}] \leftarrow \mathrm{a} 3(:,, \mathrm{i})$

10: $\mathrm{e}[\mathrm{i}] \leftarrow \mathrm{a} 4(:,:, \mathrm{i})$

11: end for

12: for $\mathrm{i}=1, . ., 3 / /$ interpolation applied on each sub-band

13: $\quad$ w1 $[\mathrm{i}]=$ interpolate $(\mathrm{b}, \mathrm{i}) / /$ using spline

14: $\quad$ w2[i] $=$ interpolate $(\mathrm{c}, \mathrm{i})$

15: $\quad$ w3 $[\mathrm{i}]=$ interpolate $(\mathrm{d}, \mathrm{i})$

16: $\quad \mathrm{w} 4[\mathrm{i}]=$ interpolate $(\mathrm{e}, \mathrm{i})$

17: end for

18: for $\mathrm{i}=1, \ldots, 3$

19: Combine the updated sub-bands into single image

20: end for using inverse $D W T$

21: calculate $P S N R$ (A, X) //finding $P S N R$ for output and 22: end original images

The proposed methodology is tested upon several renowned benchmark images and their PSNR values have been recorded. For the purpose, six different images have been considered as input, which are as shown in Figure 4. 


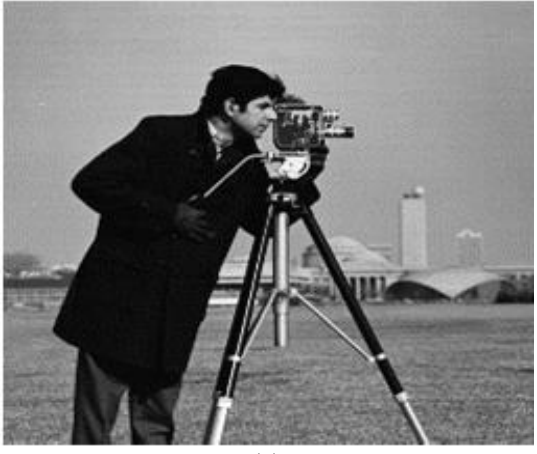

(a)

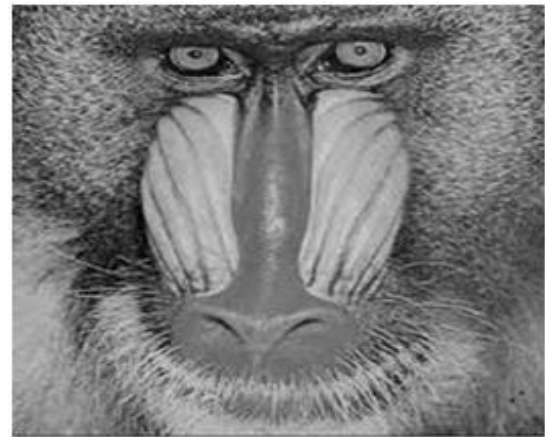

(b)

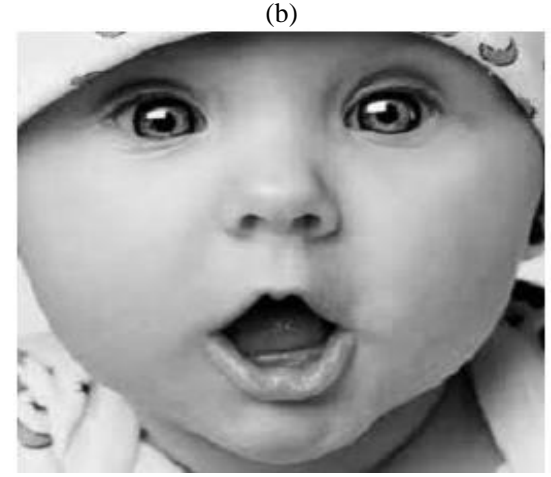

(c)

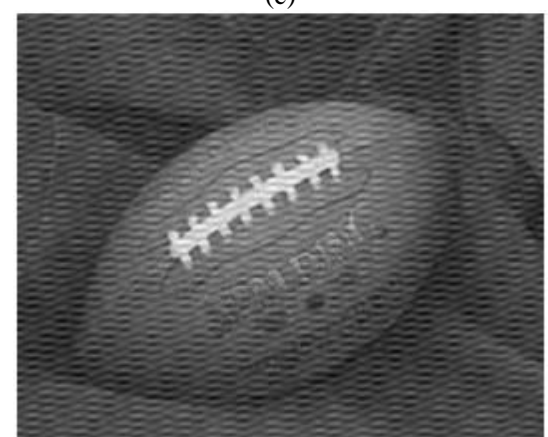

(d)



(e)
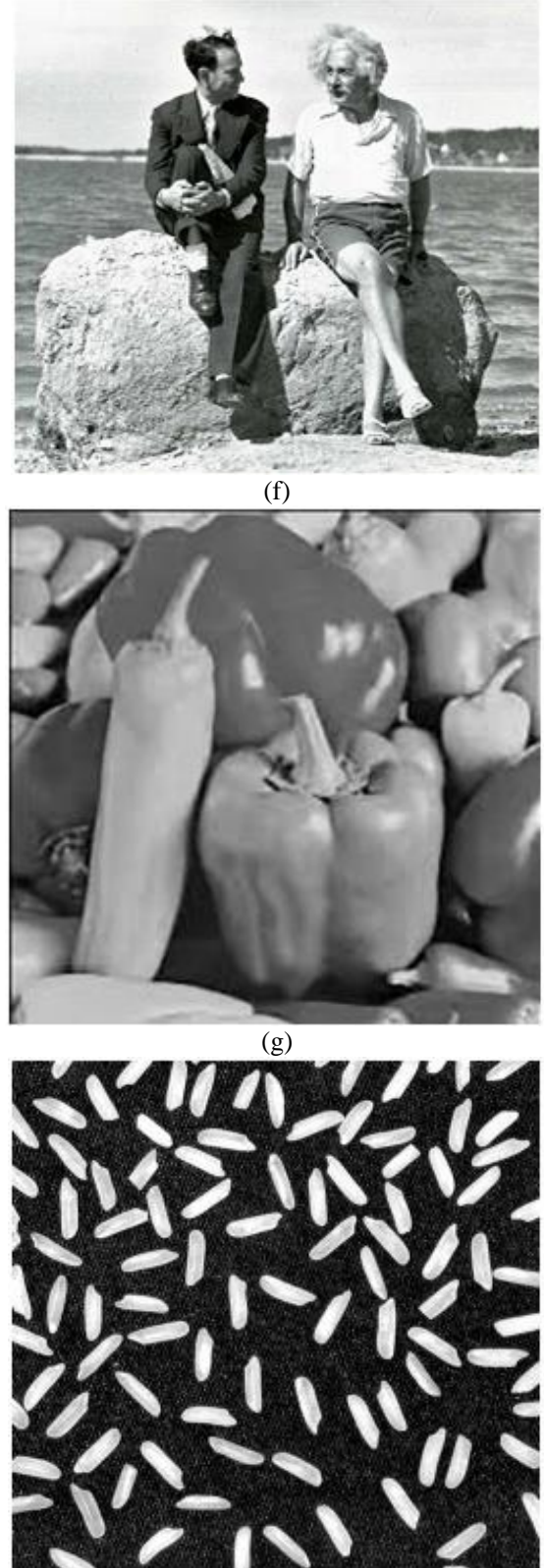

(h)

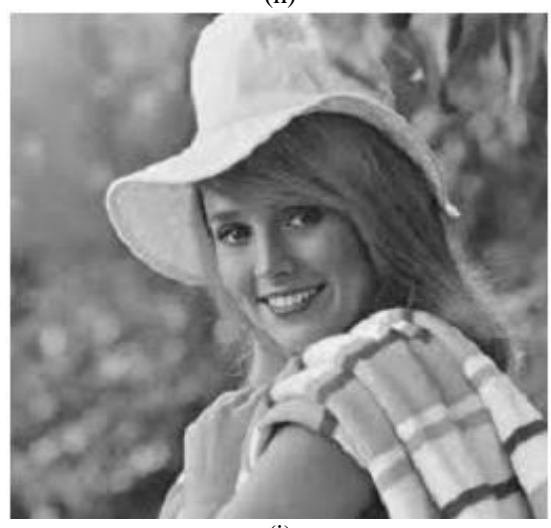

(i) 


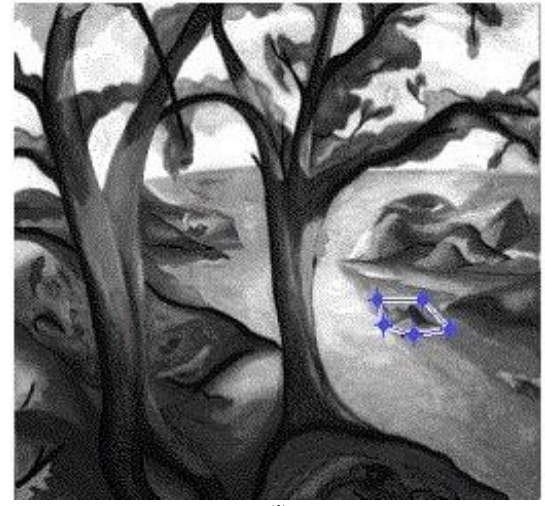

(j)

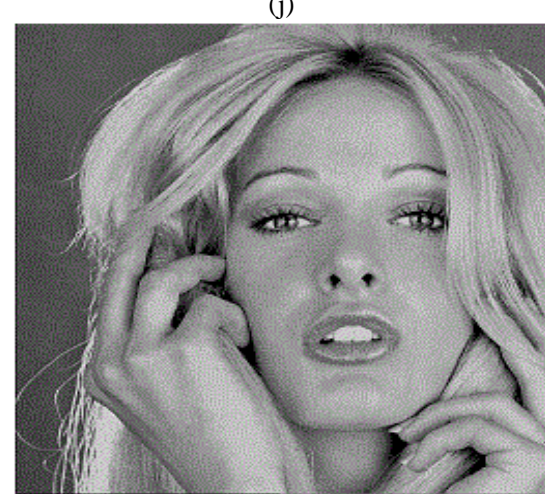

(k)

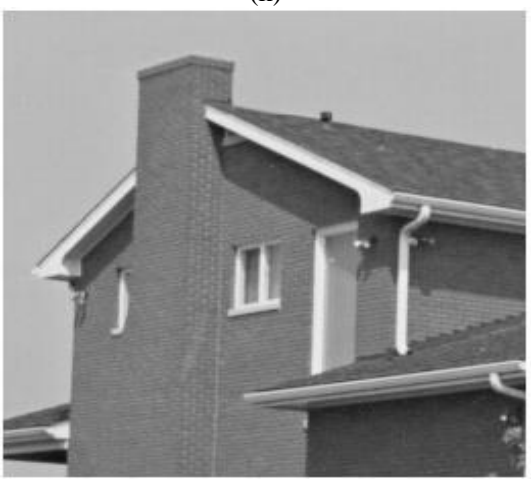

(1)

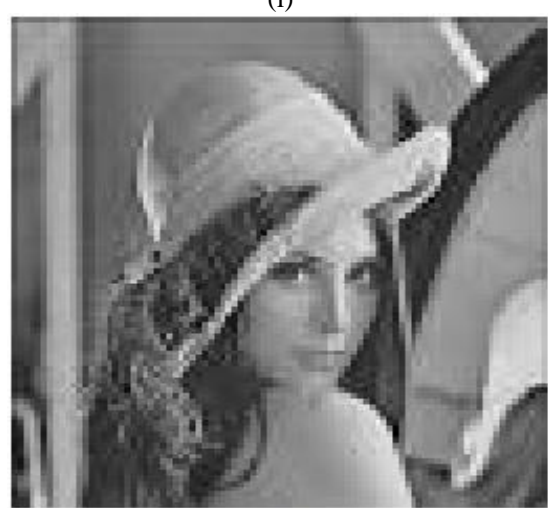

$(\mathrm{m})$

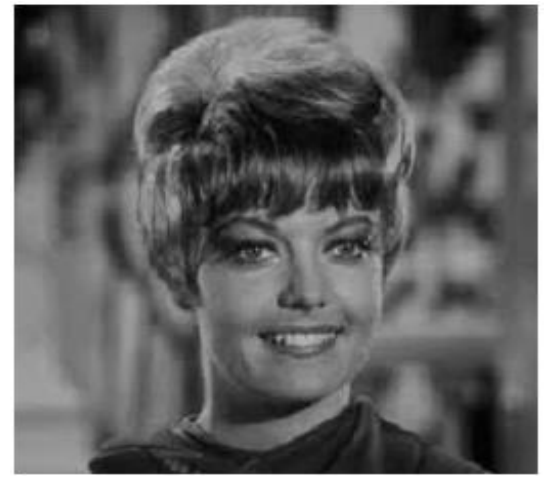

(n)

Fig.4. Original images (a) Cameraman (b) Baboon (c) Baby (d) Ball (e) Coffee (f) Men (g) Pepper (h) Rice (i) Smartg (j) Tree (k) Girl (l) House (m) Lena (n) Lady

The input images are processed and compressed using $B T C$ and the PSNR values are recorded. The results are described in Table 1 which describes the calculated values of PSNR among original and output images and their storage sizes. The output images after BTC are shown in Figure 5.

Table 1. PSNR values after applying BTC on various images

\begin{tabular}{|c|c|c|c|c|}
\hline S. No. & Image & $\begin{array}{c}\text { Input } \\
\text { Image size }\end{array}$ & $\begin{array}{c}\text { Output } \\
\text { Image size }\end{array}$ & PSNR(dB) \\
\hline 1. & Cameraman & $39.9 \mathrm{~KB}$ & $14.5 \mathrm{~KB}$ & 12.8627 \\
\hline 2. & Baboon & $31.2 \mathrm{~KB}$ & $16.9 \mathrm{~KB}$ & 13.7166 \\
\hline 3. & Baby & $16.3 \mathrm{~KB}$ & $8.65 \mathrm{~KB}$ & 20.6366 \\
\hline 4. & Ball & $24.4 \mathrm{~KB}$ & $13.7 \mathrm{~KB}$ & 16.7588 \\
\hline 5. & Coffee & $23.5 \mathrm{~KB}$ & $12.0 \mathrm{~KB}$ & 18.8555 \\
\hline 6. & Men & $34.6 \mathrm{~KB}$ & $17.0 \mathrm{~KB}$ & 11.6309 \\
\hline 7. & Pepper & $5.68 \mathrm{~KB}$ & $7.93 \mathrm{~KB}$ & 14.8992 \\
\hline 8. & Rice & $44.2 \mathrm{~KB}$ & $25.4 \mathrm{~KB}$ & 8.9885 \\
\hline 9. & Smartg & $19.8 \mathrm{~KB}$ & $10.9 \mathrm{~KB}$ & 19.8719 \\
\hline 10. & Tree & $44.1 \mathrm{~KB}$ & $21.1 \mathrm{~KB}$ & 11.3558 \\
\hline 11. & Girl & $41.3 \mathrm{~KB}$ & $20.0 \mathrm{~KB}$ & 11.5042 \\
\hline 12. & House & $18.9 \mathrm{~KB}$ & $8.75 \mathrm{~KB}$ & 18.8206 \\
\hline 13. & Lena & $23.5 \mathrm{~KB}$ & $12.2 \mathrm{~KB}$ & 16.7306 \\
\hline 14. & Lady & $173 \mathrm{~KB}$ & $9.74 \mathrm{~KB}$ & 22.1575 \\
\hline & & & & \\
\hline
\end{tabular}



(a) 


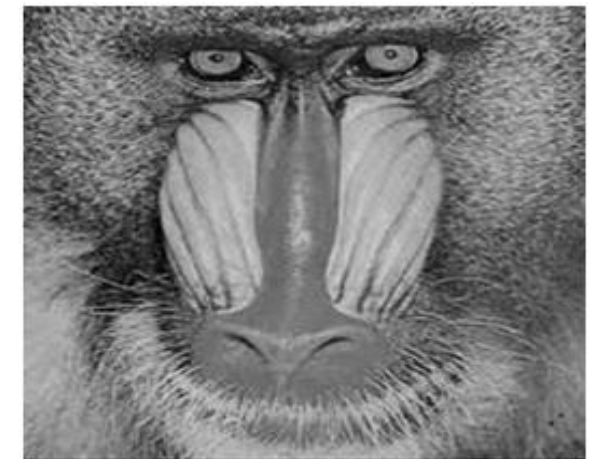

(b)



(c)

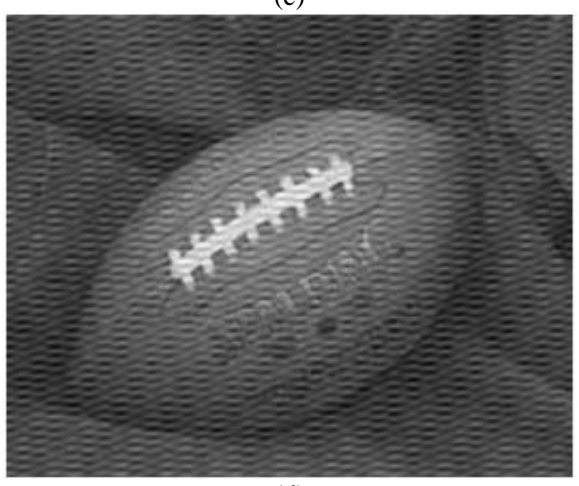

(d)

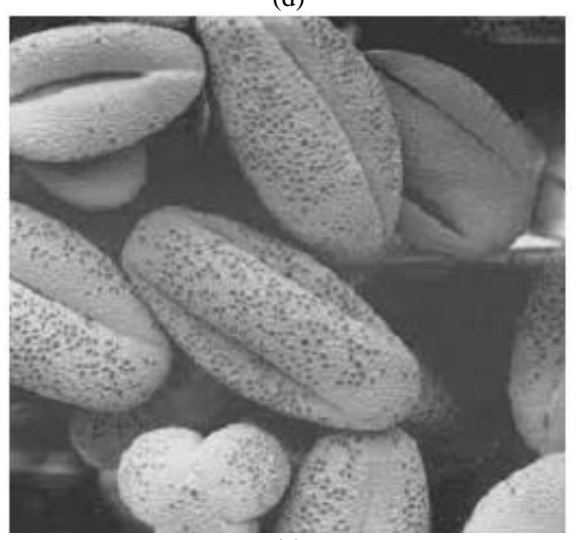

(e)


(h)

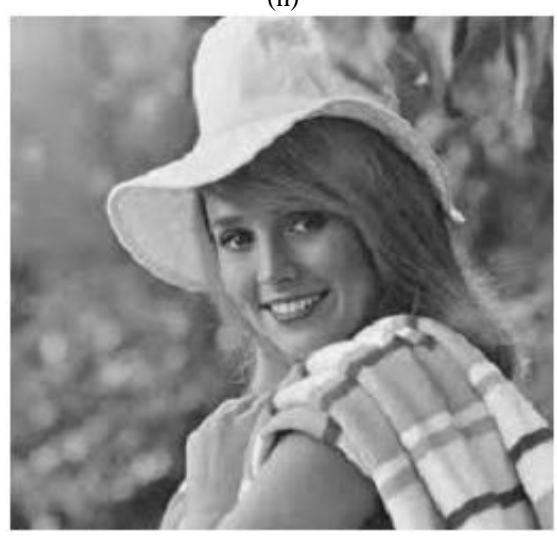

(i) 


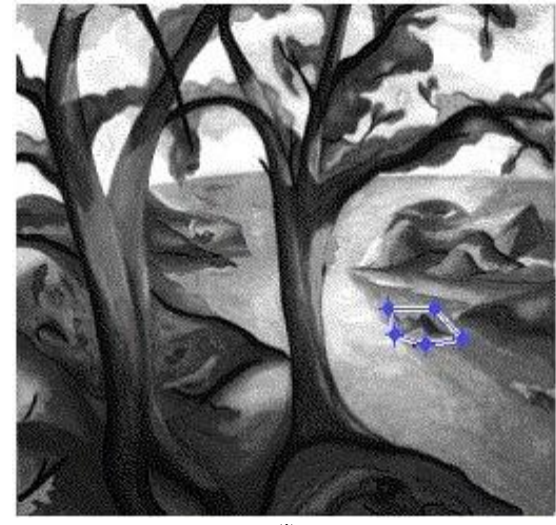

(j)

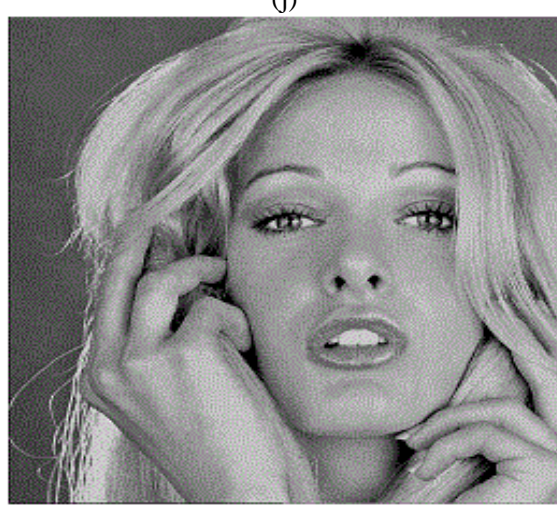

(k)



(1)



(m)



(n)

Fig.5. Compressed images (a) Cameraman (b) Baboon (c) Baby (d) Ball (e) Coffee (f) Men (g) Pepper (h) Rice (i) Smartg (j) Tree (k) Girl (l) House (m) Lena (n) Lady

The resultant images of $B T C$ provide the compressed images, but in grayscale. The decompression takes place using $D W T$ and interpolation, but for a better outcome, it considers $R G B$ images. Therefore, the resultant images are converted to $R G B$ using gray $2 r g b$ function in order to reconstruct good quality images. The converted image has values of grayscale each of $R G B$ bands. The storage size after conversion is shown in Table 2.

Table 2. Images after converting Grey Scale to RGB Scale

\begin{tabular}{|c|c|c|c|}
\hline $\begin{array}{c}\text { S. } \\
\text { No. }\end{array}$ & Images & Input Image size & Output Image size \\
\hline 1. & Cameraman & $14.5 \mathrm{~KB}$ & $16.7 \mathrm{~KB}$ \\
\hline 2. & Baboon & $16.9 \mathrm{~KB}$ & $17.5 \mathrm{~KB}$ \\
\hline 3. & Baby & $8.65 \mathrm{~KB}$ & $12.3 \mathrm{~KB}$ \\
\hline 4. & Ball & $13.7 \mathrm{~KB}$ & $14.3 \mathrm{~KB}$ \\
\hline 5. & Coffee & $12.0 \mathrm{~KB}$ & $12.6 \mathrm{~KB}$ \\
\hline 6. & Men & $17.0 \mathrm{~KB}$ & $17.8 \mathrm{~KB}$ \\
\hline 7. & Pepper & $7.93 \mathrm{~KB}$ & $11.2 \mathrm{~KB}$ \\
\hline 8. & Rice & $25.4 \mathrm{~KB}$ & $26.3 \mathrm{~KB}$ \\
\hline 9. & Smartg & $10.9 \mathrm{~KB}$ & $11.4 \mathrm{~KB}$ \\
\hline 10. & Tree & $21.1 \mathrm{~KB}$ & $23.3 \mathrm{~KB}$ \\
\hline 11. & Girl & $20.0 \mathrm{~KB}$ & $20.9 \mathrm{~KB}$ \\
\hline 12. & House & $8.75 \mathrm{~KB}$ & $9.35 \mathrm{~KB}$ \\
\hline 13. & Lena & $12.2 \mathrm{~KB}$ & $12.8 \mathrm{~KB}$ \\
\hline 14. & Lady & $9.74 \mathrm{~KB}$ & $9.98 \mathrm{~KB}$ \\
\hline
\end{tabular}

The $R G B$ images are processed using $D W T$ for enhancing its quality and is combined with spline interpolation to get the zoomed images. Firstly, the $D W T$ divides the images into 4 sub-bands and then filtering is applied to each sub-band to enhance them. The spline is used within $D W T$ to interpolate the newly formed unknown pixels. Afterwards, the inverse of $D W T$ is applied to get decompressed quality images. The outcome of images using PSNR and its storage sizes, are shown in Table 3 and the final output images are shown in Figure 6. 
Table 3. $P S N R$ values obtained using $D W T$ and interpolation

\begin{tabular}{|c|c|c|c|c|}
\hline $\begin{array}{c}\text { S. } \\
\text { No. }\end{array}$ & Image & $\begin{array}{c}\text { Input } \\
\text { Image size }\end{array}$ & $\begin{array}{c}\text { Output } \\
\text { Image size }\end{array}$ & PSNR( $\boldsymbol{d B})$ \\
\hline 1. & Cameraman & $16.7 \mathrm{~KB}$ & $53.4 \mathrm{~KB}$ & 65.2475 \\
\hline 2. & Baboon & $17.5 \mathrm{~KB}$ & $52.9 \mathrm{~KB}$ & 68.2738 \\
\hline 3. & Baby & $12.3 \mathrm{~KB}$ & $40.5 \mathrm{~KB}$ & 73.6598 \\
\hline 4. & Ball & $14.3 \mathrm{~KB}$ & $44.9 \mathrm{~KB}$ & 70.9343 \\
\hline 5. & Coffee & $12.6 \mathrm{~KB}$ & $36.6 \mathrm{~KB}$ & 72.8758 \\
\hline 6. & Men & $17.8 \mathrm{~KB}$ & $56.9 \mathrm{~KB}$ & 63.4521 \\
\hline 7. & Pepper & $11.2 \mathrm{~KB}$ & $32.1 \mathrm{~KB}$ & 71.8112 \\
\hline 8. & Rice & $26.3 \mathrm{~KB}$ & $81.5 \mathrm{~KB}$ & 72.8945 \\
\hline 9. & Smartg & $11.4 \mathrm{~KB}$ & $36.4 \mathrm{~KB}$ & 69.2453 \\
\hline 10. & Tree & $23.3 \mathrm{~KB}$ & $68.9 \mathrm{~KB}$ & 68.7463 \\
\hline 11. & Girl & $20.9 \mathrm{~KB}$ & $56.4 \mathrm{~KB}$ & 72.4289 \\
\hline 12. & House & $9.35 \mathrm{~KB}$ & $26.7 \mathrm{~KB}$ & 73.2388 \\
\hline 13. & Lena & $12.8 \mathrm{~KB}$ & $36.0 \mathrm{~KB}$ & 70.8242 \\
\hline 14. & Lady & $9.98 \mathrm{~KB}$ & $36.9 \mathrm{~KB}$ & 66.5736 \\
\hline
\end{tabular}

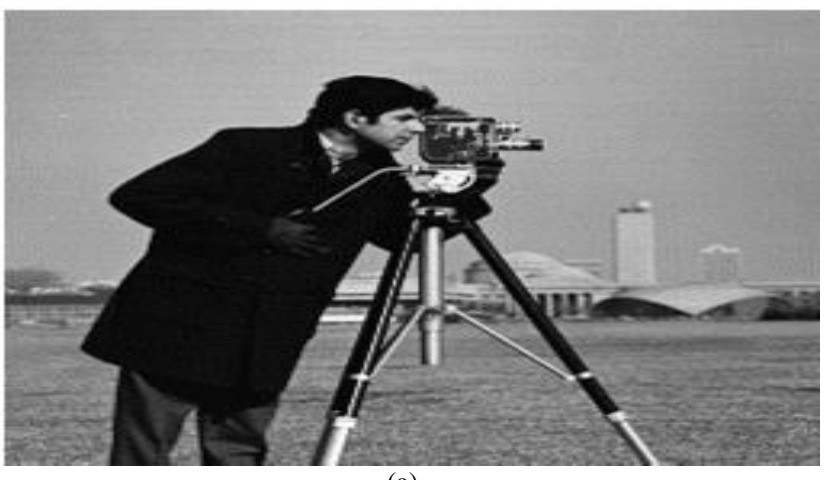

(a)

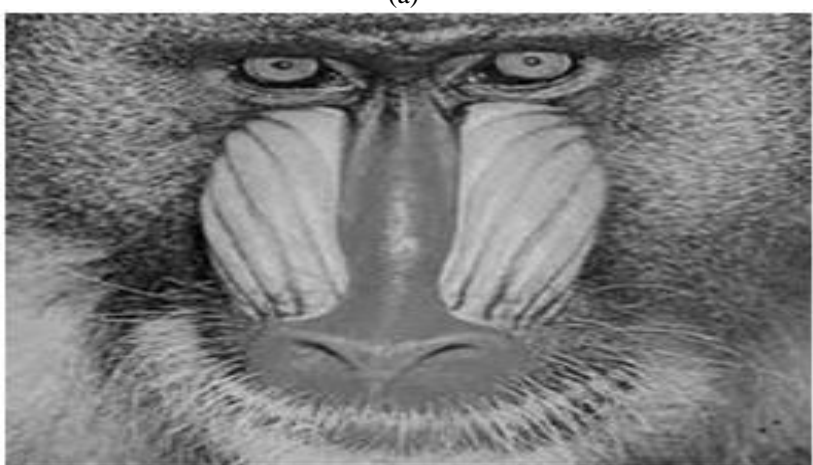

(b)
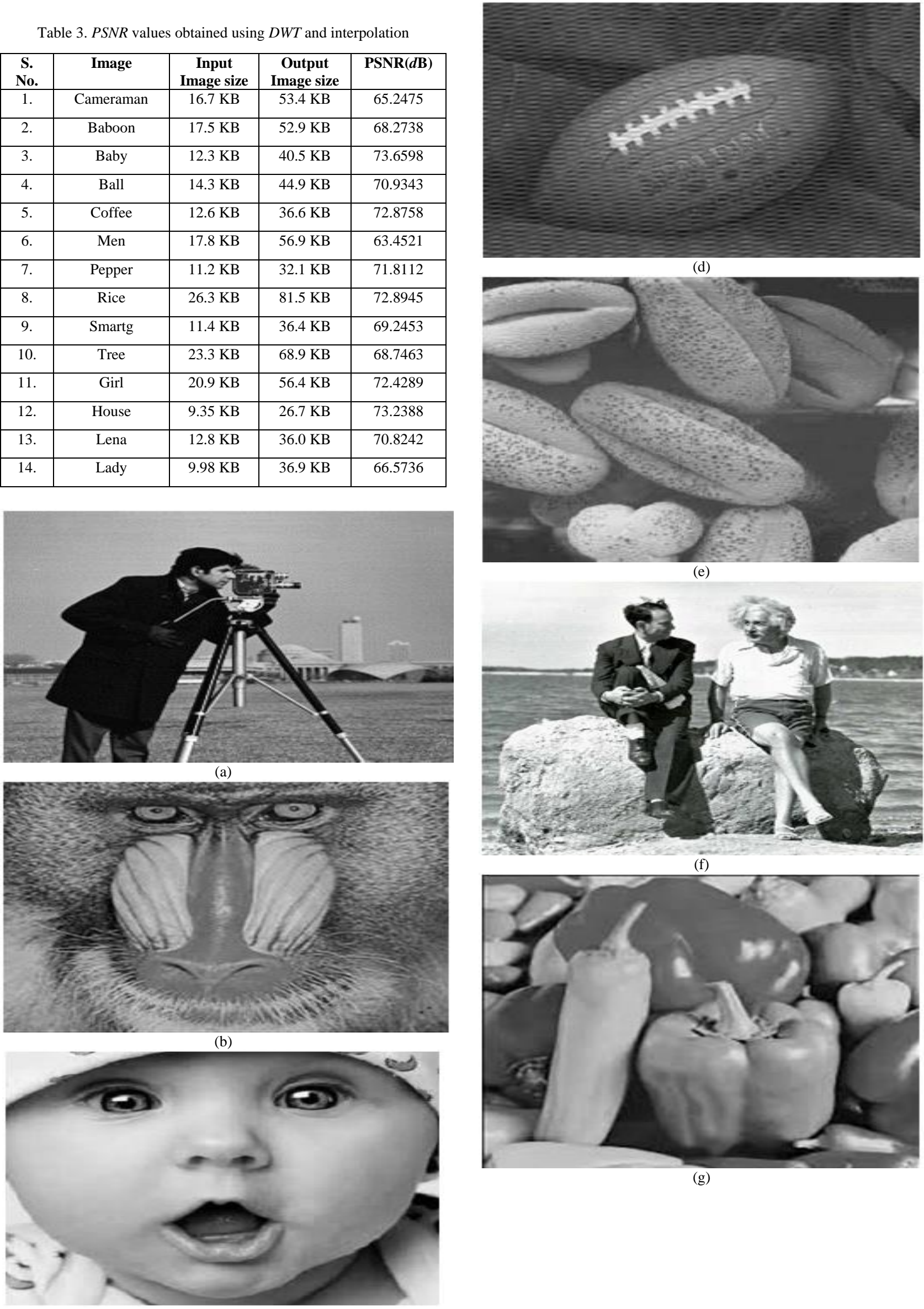

(d)



(e)
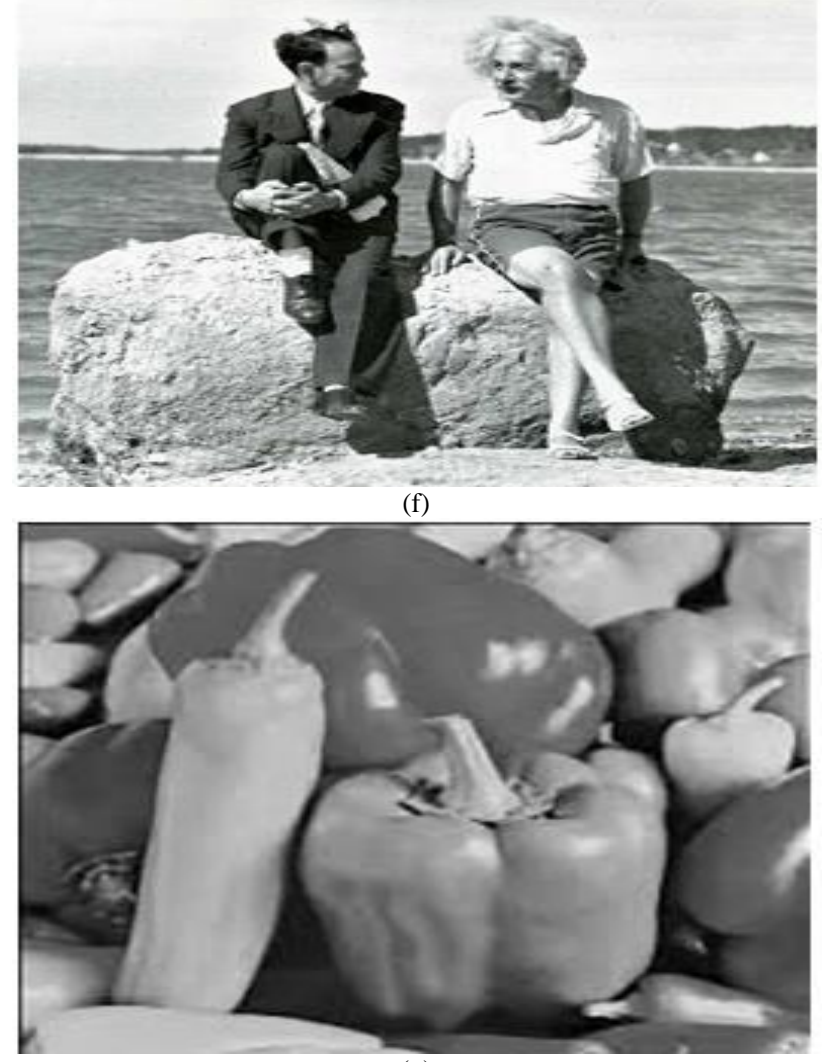

(g)

(c) 


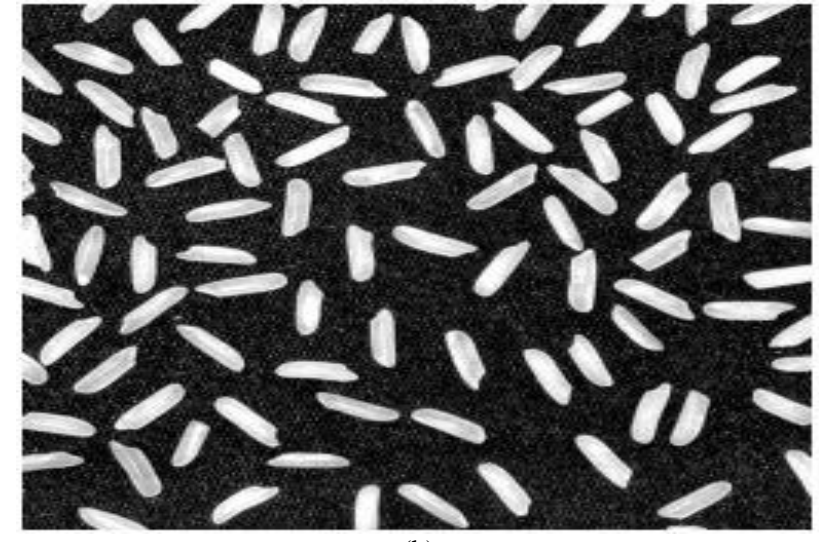

(h)

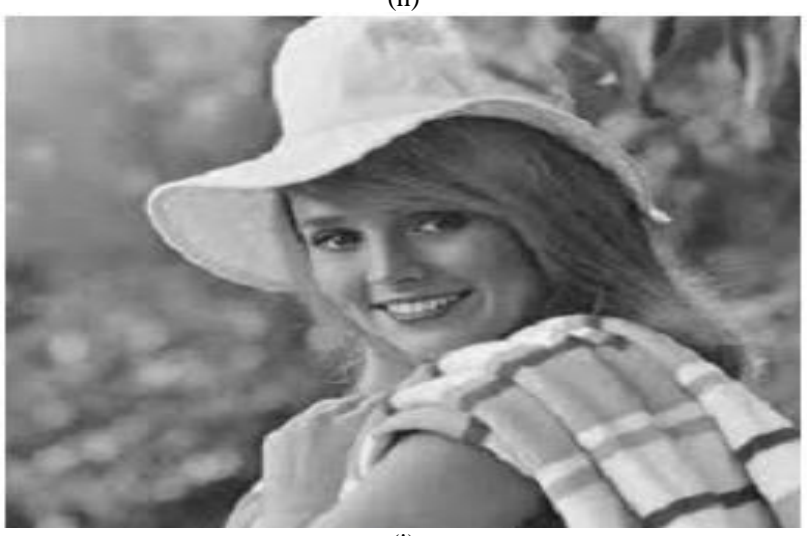

(i)

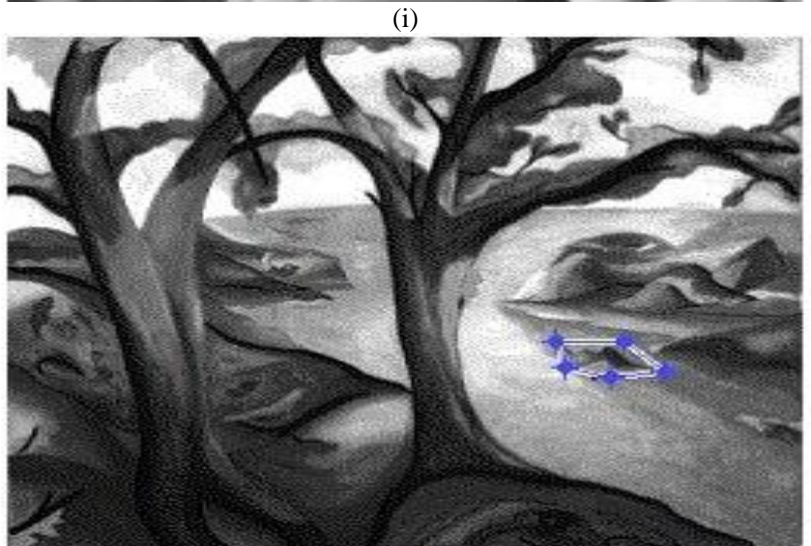

(j)

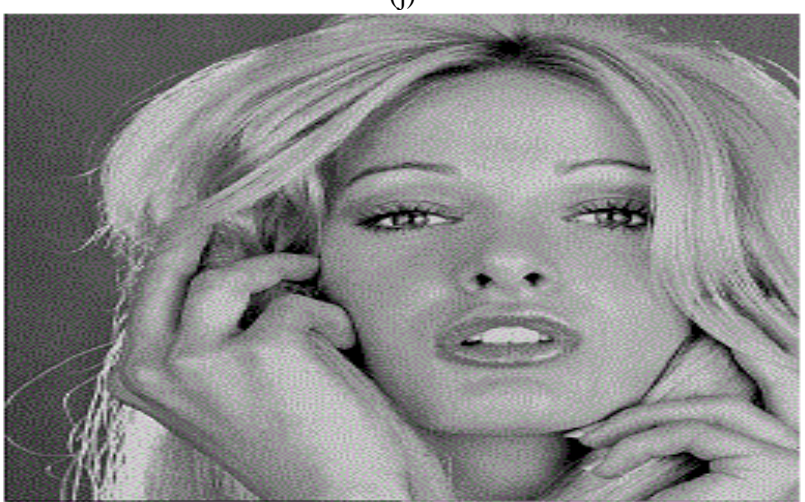

(k)

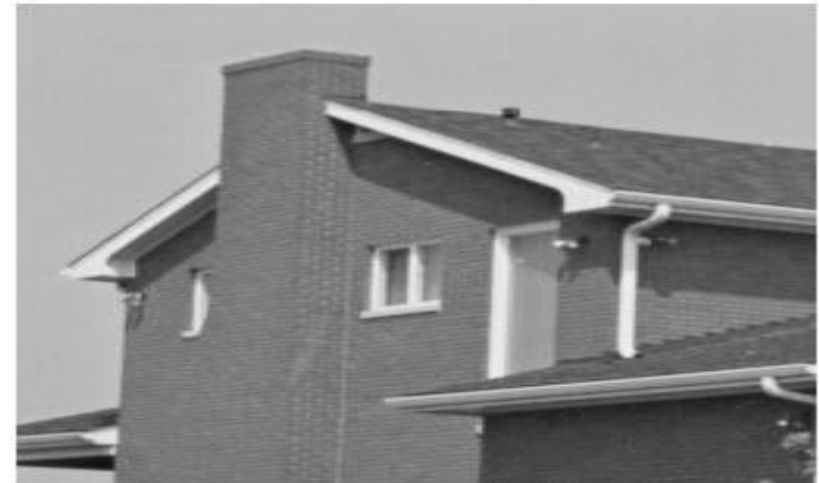

(1)

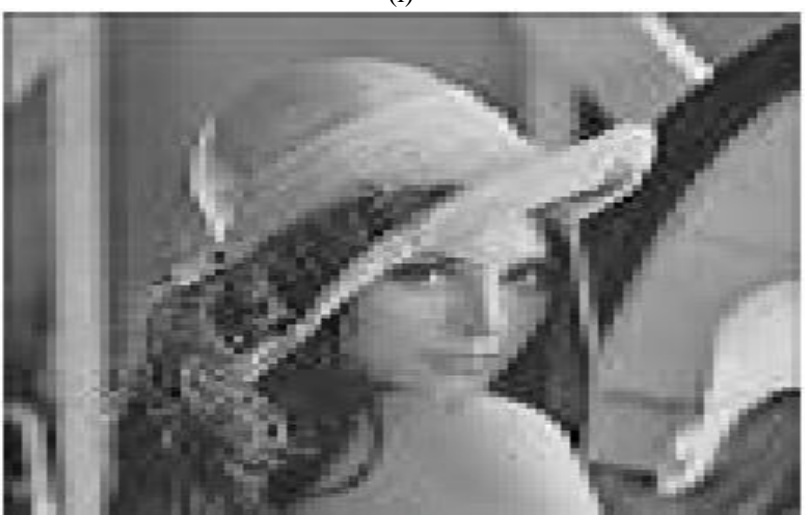

(m)

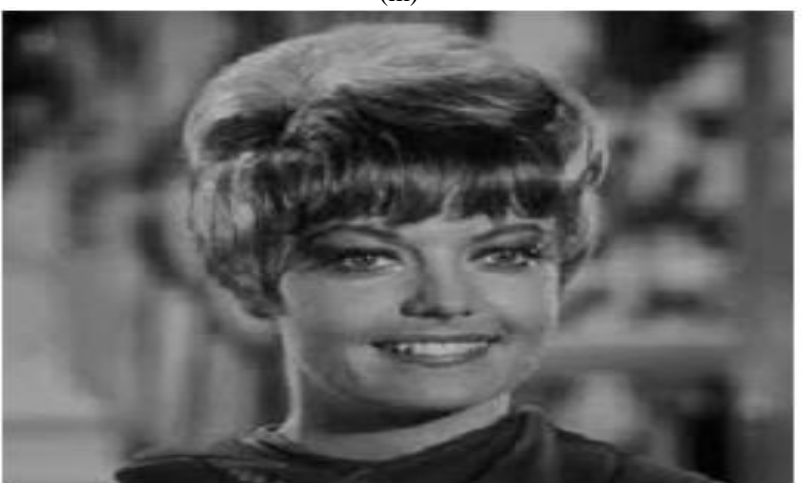

(n)

Fig.6. Reconstructed images (a) Cameraman (b) Baboon (c) Baby (d) Ball (e) Coffee (f) Men (g) Pepper (h) Rice (i) Smartg (j) Tree (k) Girl (l) House (m) Lena (n) Lady

Here the compressed images are reconstructed using wavelets and spline interpolations to get the original image which provides the information of lost pixels during compression. The PSNR values thus obtained has a minimum value of $63.4521(\mathrm{~dB})$ and the maximum value is $73.6598(\mathrm{~dB})$. This procedure is done for getting the minutest details of the compressed image and the results provides astonishing outputs in reconstructing the original image.

The input and final generated resultant images are compared on the basis of its PSNR values in order to check the improvement in decompressed images after $D W T$ and spline interpolation. The comparison is shown in Table 4. 
Table 4. Image improvement using $D W T$ and interpolation after compression $B T C$

\begin{tabular}{|c|c|c|c|}
\hline Images & $\begin{array}{c}P S N R(d \mathrm{~B}) \\
\text { values after } \\
B T C\end{array}$ & $\begin{array}{c}P S N R(d \mathrm{~B}) \\
\text { values after } \\
D W T \text { and } \\
\text { Spline } \\
\text { interpolation } \\
\end{array}$ & $\begin{array}{c}\text { Image Quality } \\
\text { Improvement } \\
\text { after } D W T \text { and } \\
\text { Spline } \\
\text { Interpolation }\end{array}$ \\
\hline Cameraman & 12.8627 & 65.2475 & $19 \%$ \\
\hline Baboon & 13.7166 & 68.2738 & $20 \%$ \\
\hline Baby & 20.6366 & 73.6598 & $28 \%$ \\
\hline Ball & 16.7588 & 70.9343 & $23 \%$ \\
\hline Coffee & 18.8555 & 72.8758 & $25 \%$ \\
\hline Men & 11.6309 & 63.4521 & $18 \%$ \\
\hline Pepper & 14.8992 & 71.8112 & $20 \%$ \\
\hline Rice & 8.9885 & 72.8945 & $12 \%$ \\
\hline Smartg & 19.8719 & 69.2453 & $28 \%$ \\
\hline Tree & 11.3558 & 68.7463 & $16 \%$ \\
\hline Girl & 11.5042 & 72.4289 & $15 \%$ \\
\hline House & 18.8206 & 73.2388 & $25 \%$ \\
\hline Lena & 16.7306 & 70.8242 & $23 \%$ \\
\hline Lady & 22.1575 & 66.5736 & $33 \%$ \\
\hline
\end{tabular}

The observed percentage of improvement has a minimum value of $12 \%$ and maximum value of $33 \%$. Thus, it is deduced that images can be compressed depending upon the user's choice and with the help of $D W T$, its quality during decompression can be improved to a certain level. Therefore, the approach is beneficial in providing a good quality image. The proposed approach is compared with existing technique in the next section.

\section{COMPARISON WITH EXISTING TECHNIQUES}

The PSNR values of proposed technique are compared with existing techniques of image processing like Nearest Neighbor Interpolation (NNI), Bi-linear Interpolation, BiCubic Interpolation, New Edge Directed Interpolation (NEDI), ICBI, Discrete Cosine Transform (DCT), Fractal Compression $(F C)$, Vector and JPEG Compression. The comparison is shown in Table 5 and Figure 7.

It is observed from the graph that the proposed technique outperforms better with the implementation of BTC, DWT and Spline interpolation. Also, the overall presentation of images is enhanced producing better quality images than the existing techniques. In comparison with the existing methods, the difference obtained is $43 \%$ of PSNR values when calculated as an average.

Table 5. Comparison of existing Techniques with Proposed Technique

\begin{tabular}{|c|c|c|c|c|c|c|c|}
\hline Images & N.N.I & Bi-linear & Bi-cubic & NEDI & ICBI & DCT & 43.0701 \\
\hline Lena & 29.4393 & 27.1202 & 27.1820 & 37.3705 & 40.9950 & 27.2109 \\
\hline Cameraman & 22.9593 & 34.1135 & 34.1628 & 39.2506 & 41.0765 & 37.7701 \\
\hline Girl & 22.9221 & 33.1643 & 33.1655 & 34.0676 & 39.0087 & 39.4769 & 24.1650 \\
\hline House & 22.9221 & 35.4563 & 35.4890 & 40.7056 & 37.0918 & 42.0016 & 25.8509 \\
\hline Pepper & 29.5585 & 33.7862 & 33.8349 & 38.1096 & 42.8506 & 41.8609 & 29.2395 \\
\hline
\end{tabular}

\begin{tabular}{|c|c|c|c|}
\hline Images & Vector compression & JPEG & Proposed Technique \\
\hline Lena & 28.2608 & 34.2701 & 70.8242 \\
\hline Cameraman & 24.1079 & 32.8705 & 65.2475 \\
\hline Girl & 23.9586 & 36.0010 & 72.4289 \\
\hline House & 29.8695 & 37.8605 & 73.2388 \\
\hline Pepper & 30.6201 & 31.0001 & 71.8112 \\
\hline
\end{tabular}




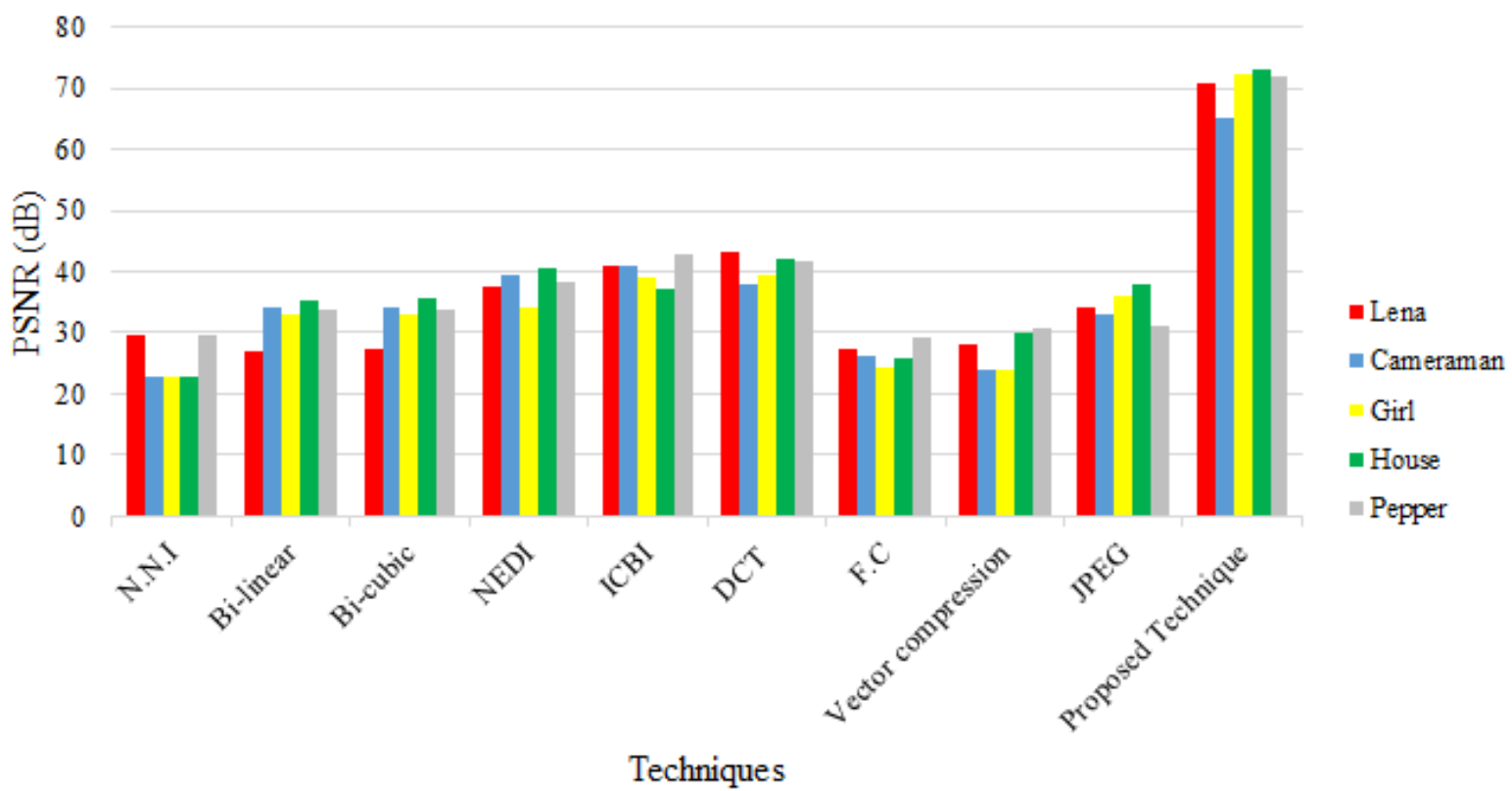

Fig.7. Comparison Graph

\section{CONCLUSION AND FUTURE SCOPE}

In this paper, a novel technique is postulated for image compression, which combines the methods of BTC and $D W T$ with spline interpolation. The observed values of $P S N R$ are appropriate and transformations have a positive impact on the visual quality of compressed images. This compression technique deals with each and every edge of the image. The method is applied as it is less complex as compare to other and easy to implement. It is observed after applying these techniques that the results produced are almost $43 \%$ better from the techniques that are used by different users for compression. These results are calculated by comparing the results of this work with existing implementations by different authors.

The proposed method can be further used in compressing and sending images among sensors deployed to get the information/data collected via images. As the sensors are capable of retaining data but to send those image data, it should be compressed to conserve energy. After sending data to the processing center, the method is applied to get decompressed images and to find the hidden information.

\section{REFERENCES}

[1] A. K. Katharotiya, S. Patel, and M. Goyani, "Comparative Analysis between DCT \& DWT Techniques of Image Compression", Journal of Information Engineering and Applications, Vol. 1, No.2, 2011.

[2] A. Kaur, and J. Kaur, "Comparison of DCT and DWT of Image Compression Techniques", International Journal of Engineering Research and Development, vol. 1, Issue 4, pp. 49-52, 2012.

[3] A. Kaushik, and M. Gupta, "Analysis of Image Compression Algorithms", International Journal of Engineering Research and Application, 2012.
[4] A. Sinha, M. Kumar, A. K. Jaiswal, and R. Sexena, "Performance Analysis Of High Resolution Images Using Interpolation Techniques in Multimedia Communication System", Signal \& Image Processing: An International Journal, Vol. 5, Issue 2,2014.

[5] B. S. Kumar, and S. Nagaraj, "Discrete and Stationary Wavelet Decomposition for Image Resolution Enhancement", International Journal of Engineering Trends and Technology (IJETT), Vol. 4, Issue 7, 2013.

[6] C. Sun, S. J. Ruan, M. C. Shie, and T. W. Pai, "Dynamic Contrast Enhancement based on Histogram Specification", IEEE Transactions on Consumer Electronics, Vol. 51, No. 4, pp.1300-1305, 2005.

[7] G. M. Padmaja, and C. H. R. Lakshmi, "Analysis of Various Image Compression Techniques", International Journal of Reviews in Computing, 2012.

[8] G. Rompani, "Warped Distance for Space Variant Linear Image Interpolation", IEEE Transactions of Image Processing, Vol. 8, Issue 5,1999.

[9] H. Huang, C. Cui, L. Cheng, Q. Liu, and J. Wang, "Grid Interpolation Algorithm Based On Nearest Neighbor Fast Search", Springer, 2012.

[10] Hitashi, and G. Kaur, and S. Sharma, "Fractal Image Compression-A Review", International Journal of Advanced Research in Computer Science and Software Engineering, Vol. 2, No. 2, 2012.

[11] M. Kaur, and G. Kaur, "A Survey of Lossless and Lossy Image Compression Technique", International Journal of Advanced Research in Computer Science and Software Engineering, Vol. 3, Issue 2, 2013.

[12] M. Tonge, P. K. Malviya, and A. Gupta, "Implementation of Digital Watermarking Algorithm based on DWT and DCT", International Journal of Advanced Engineering and Global Technology, Vol. 2, Issue 1, 2014.

[13] Md. F. Hossain, and M.R. Alsharif, "Image Enhancement Based on Logarithmic Transform Coefficient and Adaptive Histogram Equalization", IEEE International Conference on Convergence Information Technology, 2007. 
[14] R. Olivier, and C. Hanqiang, "Nearest Neighbor Value Interpolation", International Journal of Advanced Computer Science and Application", Vol. 3, Issue 4, 2012.

[15] Z. Min, W. Jiechao, L. Zhiwei and, L. Yonghua, "An Adaptive Image Zooming Method with Edge Enhancement", 3rd International Conference on Advanced Computer Theory and Engineering (ICACTE), pp. 608-611, 2010 .

\section{Authors' Profiles}

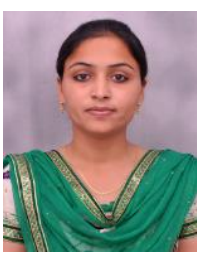

Kuldeep Mander, is M. Tech pursuing from Rayat Bahra Group of Institutes, Patiala. She is B. Tech (2014) from Baba Banda Singh Bahadur Engineering College, Fatehgarh Sahib. Her main research area of interest is Image Compression in the field of Digital Image processing.



Himanshu Jindal, is M.Tech (CSA), 2014, Ph.D. (CSE) pursuing from Thapar University, Patiala, Punjab. He has 7 international publications in reputed journals and conferences. His area of research includes Wireless Sensor Networks, Digital Image Processing, and Acoustic Communications. $\mathrm{He}$ is currently working on Underwater Acoustic Sensor Networks project funded by Thapar University, Patiala, Punjab, India.

How to cite this paper: Kuldeep Mander, Himanshu. Jindal,"An Improved Image Compression-Decompression Technique Using Block Truncation and Wavelets", International Journal of Image, Graphics and Signal Processing(IJIGSP), Vol.9, No.8, pp.17-29, 2017.DOI: 10.5815/ijigsp.2017.08.03 